\title{
RM07
}

\section{Lies, Damned Lies and a Grain of Truth}

\section{Daly* (Schlumberger)}

\section{SUMMARY}

Modeling of reservoirs has become more mathematically sophisticated over the last twenty years. In particular stochastic models have become the norm in very many, if not most reservoir studies. The reasons for this are threefold

1) The advance of stochastic reservoir modeling as a science exposed the shortcomings implicit in the older deterministic models, namely underestimation of the role of heterogeneity.

2) Stochastic methods provide the best mechanism to incorporate various data types into a model.

3) They allow for some understanding and modeling of the uncertainty present in the reservoir.

We have arrived at a point in time where many of the standard techniques for modelling are available in all of the principal commercial software packages (which is not to say that the methods cannot and should not be improved further). It may therefore be a good time to ask the wider question about how they are being used in practice particularly with regard to the major issue of understanding risk.

In this presentation we look at how closed mathematical models, while being a fundamental building block, do not lead, on their own, to a good methodology for understanding risk. An analogy with the mathematisation of risk in banking and the credit markets is noted. The conclusion, which is very obvious, is to increase the amount of speculative geological model building and peer to peer discussion about scenarios. However, the current practice of reservoir modeling provides stumbling blocks making users reluctant, or even unable to easily explore differing scenarios. Indeed this difficulty extends beyond heterogeneity modeling into geophysics, petrophysics etc. To change this is a big task, which we don't pretend to have resolved here, but by shining light on the problem, and by making a small suggestion to improve matters for heterogeneity modeling, we hope to open discussion about the subject. 
Modeling of reservoirs has become more mathematically sophisticated over the last twenty years. In particular stochastic models have become the norm in very many, if not most reservoir studies. The reasons for this are threefold

1) The advance of stochastic reservoir modeling as a science exposed the shortcomings implicit in the older deterministic models, namely underestimation of the role of heterogeneity.

2) Stochastic methods provide the best mechanism to incorporate various data types into a model.

3) They allow for some understanding and modeling of the uncertainty present in the reservoir.

We have arrived at a point in time where many of the standard techniques for modelling are available in all of the principal commercial software packages (which is not to say that the methods cannot and should not be improved further). It may therefore be a good time to ask the wider question about how they are being used in practice particularly with regard to the major issue of understanding risk.

In this presentation we look at how closed mathematical models, while being a fundamental building block, do not lead, on their own, to a good methodology for understanding risk. An analogy with the mathematisation of risk in banking and the credit markets is noted. The conclusion, which is very obvious, is to increase the amount of speculative geological model building and peer to peer discussion about scenarios. However, the current practice of reservoir modeling provides stumbling blocks making users reluctant, or even unable to easily explore differing scenarios. Indeed this difficulty extends beyond heterogeneity modeling into geophysics, petrophysics etc. To change this is a big task, which we don't pretend to have resolved here, but by shining light on the problem, and by making a small suggestion to improve matters for heterogeneity modeling, we hope to open discussion about the subject. 